\title{
How Children Learn to Speak
}

\author{
Asfar Sadath K. \\ Assistant Professor \\ Sullamussalam Science College \\ Malappuram, Kerala, India \\ ashfar.clt@gmail.com
}

Abstract

Children acquire language spontaneously without being explicitly taught how. Their mastery of sounds passes through stages determined by a progression from unmarked (ontologically primary) to more marked sounds, unmarked ones also being those most commonly found in languages and least likely to erode over time, as discovered by founding linguist Roman Jakobson. Their mastery of other aspects of grammar proceeds along with their ability to master rules rather than simply memorize.

Article

It was once thought that children learned to speak simply by imitating adults: They hear adults saying words, and then they pick up words. However, modern linguists have found that it is something apparently innate to the species, and thus it proceeds according to basic aspects of linguistic structure. Children do not just master a basket of words and expressions. There's something more constrained and systematic going on. Speaking ability merges without teaching, like fish swimming. Many cultures do not "teach" language, and much of the language is not taught (one might correct The cat's hands are dirty but not Where kitty go?). 
In general, we only teach so much, and we cannot correct everything. Children often insist that what they are saying is right and eventually learn to speak correctly on their own. Babbling is universal: The first stage of language acquisition is spontaneous vocalizing. Children worldwide do this. Even deaf kids do it, and kids with throat blockages do it after their throats are unblocked.

Learning to make sounds comes before learning to make words, and the order in which sounds are learned follows a pattern. At first, voiced stops and nasals predominate; fricatives and liquids are rare. That is, children learn the most unmarked sounds first. By age two, a child has all consonants except $/ \mathrm{j} /$ and $/ \square /$.

Children's first renditions of sounds are unmarked substitutions.

1. Rendering fricatives as stops: sing as ting, zebra as dibra.

2. Fronting, e.g., alveopalatal to alveolar: shoes becomes sooz, velar to alveolar: goat becomes doat.

3. Voicing, e.g., of stops before vowels (pot to baht).

4. Denasalization (jam to dab, room to woob). (Note the effects piling on one another, which is what makes children hard to understand.)

Roman Jakobson and other scholars who developed the concept of markedness are known as the Prague School. Roman Jakobson (1896-1982) was a Russian thinker who observed, during a series of exchanges with fellow linguist Nikolai Trubetskoy (1890-1938), that the order in which children learn sounds demonstrates that sounds are related to one another in a hierarchy based on markedness.

All children, learning all languages, acquire the vowel /a/ first, then stop consonants, and then nasal consonants before others.

This implies that there is something developmentally primary about those sounds. Babies do not master them first because they are the easiest to make, given that during the 
initial babbling stage, children make all sounds. Rather, /a/ and stops and nasals are the easiest for the brain to perceive as distinct sounds.

These sounds are the least marked. Bilabial fricatives like //3/ or / $\square /$ or vowels like / $\square /$ are more marked compared to these. No child masters the latter sounds first.

That some sounds are especially unmarked is supported by the fact that while some languages have very small inventories of phonemes, even the ones with the fewest sounds have stops and /a/. No languages have, instead, only nasals and glides.

How children acquire morphology shows something else that is interesting.

First, children retain fossilized memorization of irregular forms: came, men.

Then children pass through a stage of processing that there are rules in the language but of overgeneralization, in which they produce forms like feets and mouses, whichironically — shows that they are internalizing rules. Then the exceptions are "relearned."

The process of acquiring syntax is much more gradual than for morphology. Oneword stage (at one year old): At first, children use single words to refer to entire propositions: “dada" means Here comes Daddy or This shoe is Daddy's, etc.

Two-word stage (at one year and eight months): children refer to an entity and a predicate ("dada chair," "hit doggie"), which is the heart of the configuration of the syntactic trees we encountered in earlier lectures.

Telegraphic stage (between two- and two-and-a-half years old): word order is accurate, but no grammatical items such as definite articles and the verb to be: "Daddy like this book," "I good boy."

They first understand the concrete, rather than the words that express abstract concepts. This correlates with how in our adult consciousness concrete words become 
grammatical ones "under the radar," such as going to in the literal sense of movement becoming a marker of futurity as in I am going to think about that someday.

How children acquire vocabulary also follows an orderly progression.

A. Overextension is very common—fly may refer to dirt, dust, small bugs, toes, crumbs; quack to birds, flies, even coins; dog to any animal. Underextension occurs as well, such as kitty referring only to the cat in the house.

B. By one year and eight months old, children typically know about 50 words. By age five, children are learning about 15 or 20 words per day (about a word every two hours), and by age eight they know about 18,000 basic words.

C. It is often said that a child's basic acquisition of a language is largely complete at six or seven.

That certainly seems to be true of children mastering European languages such as English, French, and German. However, there are anecdotal reports that children do take a little bit longer to learn languages that are extremely complex. The Cree Native American language, which is spoken in Canada, is massively complex, and it has been said that children are not competent in even the basic language until they are about 10 years old. 


\section{Bibliography}

Tomasello, Michael, N. Akhtar, K. Dodson, and L. Rekau. 1997. Differential productivity in young children's use of nouns and verbs. Journal of Child Language 24, 373-87.

Pinker, Steven. 1984. Language learnability and language development. Cambridge, MA: Harvard University Press.

O’Grady, William. 1997. Syntactic development. Chicago: University of Chicago Press. 